\title{
ACCOUNTS
}

of chemical research

\section{D Electron Microscopy: Principles and Applications}

\author{
DAVID J. FLANNIGAN ${ }^{\dagger}$ AND AHMED H. ZEWAIL* \\ Physical Biology Center for Ultrafast Science and Technology, \\ Arthur Amos Noyes Laboratory of Chemical Physics, California Institute \\ of Technology, Pasadena, California 91125, United States
}

RECEIVED ON JUNE 1, 2012

\section{CONSPECTUS}

$\mathrm{T}$ he transmission electron microscope (TEM) is a powerful tool enabling the visualization of atoms with length scales smaller than the Bohr radius at a factor of only 20 larger than the relativistic electron wavelength of $2.5 \mathrm{pm}$ at $200 \mathrm{keV}$. The ability to visualize matter at these scales in a TEM is largely due to the efforts made in correcting for the imperfections in the lens systems which introduce aberrations and ultimately limit the achievable spatial resolution. In addition to the progress made in increasing the spatial resolution, the TEM has become an all-in-one characterization tool. Indeed, most of the properties of a material can be directly mapped in the TEM, including the composition, structure, bonding, morphology, and defects. The scope of applications spans essentially all of the physical sciences and includes biology.

Until recently, however, high resolution visualization of structural changes occurring on sub-millisecond time scales was not possible. In order to reach the ultrashort temporal domain within which fundamental atomic motions take place, while simultaneously retaining high spatial resolution, an entirely new approach from that of millisecond-limited TEM cameras had to be conceived. As shown below, the approach is also different from that of nanosecond-limited TEM, whose resolution cannot offer the ultrafast regimes of dynamics. For this reason "ultrafast electron microscopy" is reserved for the field which is concerned with femtosecond to picosecond resolution capability of structural dynamics.

In conventional TEMs, electrons are produced by heating a source or by applying a strong extraction field. Both methods result in the stochastic emission of electrons, with no control over temporal spacing or relative arrival time at the specimen. The timing issue can be overcome by exploiting the photoelectric effect and using pulsed lasers to generate precisely timed electron packets of ultrashort duration. The spatial and temporal resolutions achievable with short intense pulses containing a large number of electrons, however, are limited to tens of nanometers and nanoseconds, respectively. This is because Coulomb repulsion is significant in such a pulse, and the electrons spread in space and time, thus limiting the beam coherence. It is therefore not possible to image the ultrafast elementary dynamics of complex transformations. The challenge was to retain the high spatial resolution of a conventional TEM while simultaneously enabling the temporal resolution required to visualize atomic-scale motions.

In this Account, we discuss the development of four-dimensional ultrafast electron microscopy (4D UEM) and summarize techniques and applications that illustrate the power of the approach. In UEM, images are obtained either stroboscopically with coherent single-electron packets or with a single electron bunch. Coulomb repulsion is absent under the single-electron condition, thus permitting imaging, diffraction, and spectroscopy, all with high spatiotemporal resolution, the atomic scale (sub-nanometer and femtosecond). The time resolution is limited only by the laser pulse duration and energy carried by the electron packets; the CCD camera has no bearing on the temporal resolution. In the regime of single pulses of electrons, the temporal resolution of picoseconds can be attained when hundreds of electrons are in the bunch.

The applications given here are selected to highlight phenomena of different length and time scales, from atomic motions during structural dynamics to phase transitions and nanomechanical oscillations. We conclude with a brief discussion of emerging methods, which include scanning ultrafast electron microscopy (S-UEM), scanning transmission ultrafast electron microscopy (STUEM) with convergent beams, and time-resolved imaging of biological structures at ambient conditions with environmental cells. 


\section{Introduction}

In the last century, our ability to determine atomic-scale structures of organic, inorganic, and biological systems has dramatically improved. Indeed, in the past one hundred years, the state-of-the-art in structure determination has gone from Bragg's original work on simple salts and metals such as copper ${ }^{1}$ to recent visualization of the 605 subunit of the eukaryotic ribosome, a complex system comprised of some 5000 nucleotides and nearly 50 proteins. $^{2}$ These advances in structure determination can be attributed, at least in part, to the development of bright, electron and hard $X$-ray sources and to the exponential rise in computing power. The phrase "seeing is believing" is a good descriptor of the powerful impact atomic-scale static structure determination has had on our understanding of matter, as demonstrated by the number of Nobel Prizes awarded for works that focused and relied on the use of such techniques.

While structure determination is undeniably powerful, the multitude of pathways available to a complex system on its potential energy surface, due to the enormous number of degrees of freedom, precludes the formulation of a complete description of its function from such static images. For example, while the static structures of many proteins have been determined, we still have a limited understanding of their fundamental dynamical processes such as folding and molecular recognition. Similarly, for strongly correlated materials having a variety of emergent properties (e.g., superconductivity, ferroelectricity, etc.), there exist unique kinetic pathways, and the ability to control the trajectory of such systems in order to bring about desired effects is of keen interest. Therefore, visualization of structures, with the appropriate spatiotemporal resolutions, as they transform from one thermodynamically stable state to the next, is critical to our understanding of matter's behavior.

Relativistic ultrashort pulses of electrons accelerated to near the speed of light are ideal for probing transient and intermediate structures with atomic-scale resolution. This is because the relativistic wavelength of these particles is on the order of a picometer, which is a small fraction of interatomic separations. The electron-atom interaction is strong, having a scattering cross section roughly $10^{6}$ times larger than that of an X-ray photon. ${ }^{3,4}$ This strong interaction allows for the generation of diffraction patterns and images having a large signal-to-noise ratio from a relatively low number of electrons. Furthermore, because electrons are charged particles, they are easily deflected and focused with table-top magnetostatic and electrostatic lens arrays, such as one finds in a transmission electron microscope (TEM). Indeed, electron-based imaging has had an impact in nearly every area of research. By generating and controlling ultrashort electron packets, the fourth dimension of time thus enables space-time visualization of transient and intermediate structures of molecules, crystals, and complex systems.

In the 1980s, the development of the field of femtochemistry made possible observation of molecular transformations occurring during the course of chemical reactions. ${ }^{5}$ The ultrafast spectroscopic techniques that were developed reveal the temporal evolution of simple and complex systems, but elucidate structures of only relatively small molecules. For more complex structures, a new approach was needed. At Caltech, the method of choice was, and still is, ultrafast electron microscopy (UEM) ${ }^{6,7}$ whose development began in 1999 with diffraction and crystallography. ${ }^{8,9}$

In this Account, we highlight the principles involved and focus on recent applications of the UEM approach in materials and biological science. The intention is not to give an exhaustive literature survey as a recent book and review contains the relevant references. ${ }^{6,7}$ We also illustrate the power of several variants of UEM, in addition to the workhorse parallel-beam geometry, and these include (a) convergent-beam imaging and diffraction (CB-UEM) ${ }^{10}$ (b) photon-induced near-field electron microscopy (PINEM) ${ }^{11}$ (c) 4D tomographic imaging, ${ }^{12}$ (d) femtosecond electronenergy loss spectroscopy (FEELS), ${ }^{13}$ and (e) 4D Lorentz microscopy. ${ }^{14}$ With these and other variants, scanning ultrafast electron microscopy (S-UEM) ${ }^{15}$ and scanning transmission ultrafast electron microscopy (ST-UEM), ${ }^{16}$ we give an account of the elementary processes that can be studied, from chemical bonding and reactivity to phase transitions and nanomechanical properties of complex systems.

\section{UEM Principles}

Before discussing the applications of UEM, we provide a description of some defining principles of the method. In order to resolve the fleeting structures associated with transient phenomena, the effective shutter rate of UEM has to be on the order of the rate of change of the transformation in the system of interest. ${ }^{17} \mathrm{~A}$ simple analogy is that of flash photography; if the motion of the object is faster than the shutter, the resulting image is blurred. In addition to shutter rate, the number of photons hitting the detector is a key component to image formation. At fixed sensitivity, the number of photons striking the detector should be enough to form an image, within 
which the amount of information contained is dependent on the signal-to-noise ratio.

In UEM, we use the concept of relativistic electron stroboscopy to resolve ultrafast atomic-scale structural dynamics. Thus, visualizing structural dynamics by acquiring a series of snapshots of the system demands the marriage of ultrafast probing techniques with those of structural determination by microscopy and diffraction, as well as the development of concepts describing the simultaneous temporal and spatial resolutions and coherences. If the transformation is irreversible, a single-pulse frame recording is required, and this too has been achieved in UEM.

Typically, the structural change is initiated either by rapid heating ( $\mathrm{T}$-jump) of the sample through coherent electronic excitation induced by femtosecond laser pulses or with a picosecond (nanosecond) optical pulse. Electrons comprising a probing pulse, which are generated via the photoelectric effect, are accelerated to some fraction of the speed of light ( $0.7 c$ for $200 \mathrm{keV}$ ) and arrive at the sample at some well-defined time after the heating excitation pulse, or the T-jump pulse. For processes involving structural changes that are not reversible on the experimental time scale, a single probing pulse contains enough electrons to form an image. Because electrons are fermions, however, each particle in the dense bunch will experience significant Coulomb repulsion. Thus, the temporal resolution of this approach was limited to the fast domain of nanoseconds. ${ }^{18}$

In order to reach the femtosecond ${ }^{19,20}$ and perhaps attosecond domains, ${ }^{21,22}$ the negative effects of Coulomb repulsion had to be circumvented. We achieved this by using timed, coherent single-electron packets. ${ }^{7,23}$ By varying the time between the heating pulses to be commensurate with the time of change of the specimen relaxation to the initial, ground state, structure, images can be built up by using repeated single-electron packets at a fixed delay relative to the excitation pulse. Once the image is formed, the delay can be changed with femtosecond precision, and a series of images for the structure can be generated in the same manner. In this way, we obtain diffraction patterns, images, and electron energy spectra, all with good spatiotemporal resolutions.

\section{UEM Applications}

(Sub)Single-Particle Imaging. Electron energy-loss spectroscopy (EELS), a powerful analytical tool, takes advantage of inelastic scattering processes caused by electron excitation of valence and core-level transitions. In the photoninduced near-field (PINEM) ${ }^{11}$ variant of UEM, photons are used for the excitation instead of electrons. In this way, the photons create near-field evanescent fields in the nanostructure studied and the probing electron packet images these fields, which extend only to nanometers in space and survive temporally for femtoseconds. Indeed, the imaging and spectroscopy methods of PINEM, and the ability to perform single and (sub)single-particle imaging (Figure 1), rely on the strong interaction between photons and electrons, as facilitated by the presence of a nanostructure.

A striking demonstration of PINEM is shown by the singleparticle imaging of evanescent fields surrounding nanotubes. As shown in Figure $1 \mathrm{~A}$, in addition to imaging these fields in real-space, with PINEM we have access to the ultrafast temporal domain with femtosecond resolution. These energy-filtered UEM images were acquired by using only electrons that have gained quantized photon energy relative to the main beam, thus revealing the spatial region where the electrons and photons have coupled. As can be directly observed from the images, the contrast increases and decreases in strength in less than $1 \mathrm{ps}$.

One significant example of this methodology was the application of PINEM to the imaging of biological structures. ${ }^{24}$ Shown in Figure 1B are polarization-dependent and time-resolved PINEM images of protein vesicles. We note that the optical properties of biological structures and biomaterials differ greatly from inorganic semiconductors and metals (in their electron scattering and sensitivity to radiation damage), yet PINEM is still possible and robust, thus suggesting the applicability to a wide range of systems. ${ }^{25}$ For example, because protein vesicles are transparent to the laser excitation wavelength, high fluences could be used to generate strong PINEM signals. Further, because imaging of evanescent fields results in a strengthening of the contrast, the spatial location of the enhancement can be controlled via laser polarization, time scale, and tomographic tilting.

A significant advancement made to the PINEM method was recently demonstrated by employing nanometer-sized scanning electron probes to map the field of a single particle or an interface while optically excited (Figure 1C). ${ }^{26}$ Spectrum images were obtained for nanoscale dielectric fields surrounding individual metal nanoparticles and extended interfaces. Importantly, the size of the scanned electron probe relative to the nanoparticle dimensions allowed for a spot-by-spot mapping of the fields inside and outside the particle. This convergent-beam PINEM development thus combines the high spatial resolution of electron microscopy with the high energy resolution of optical techniques while 


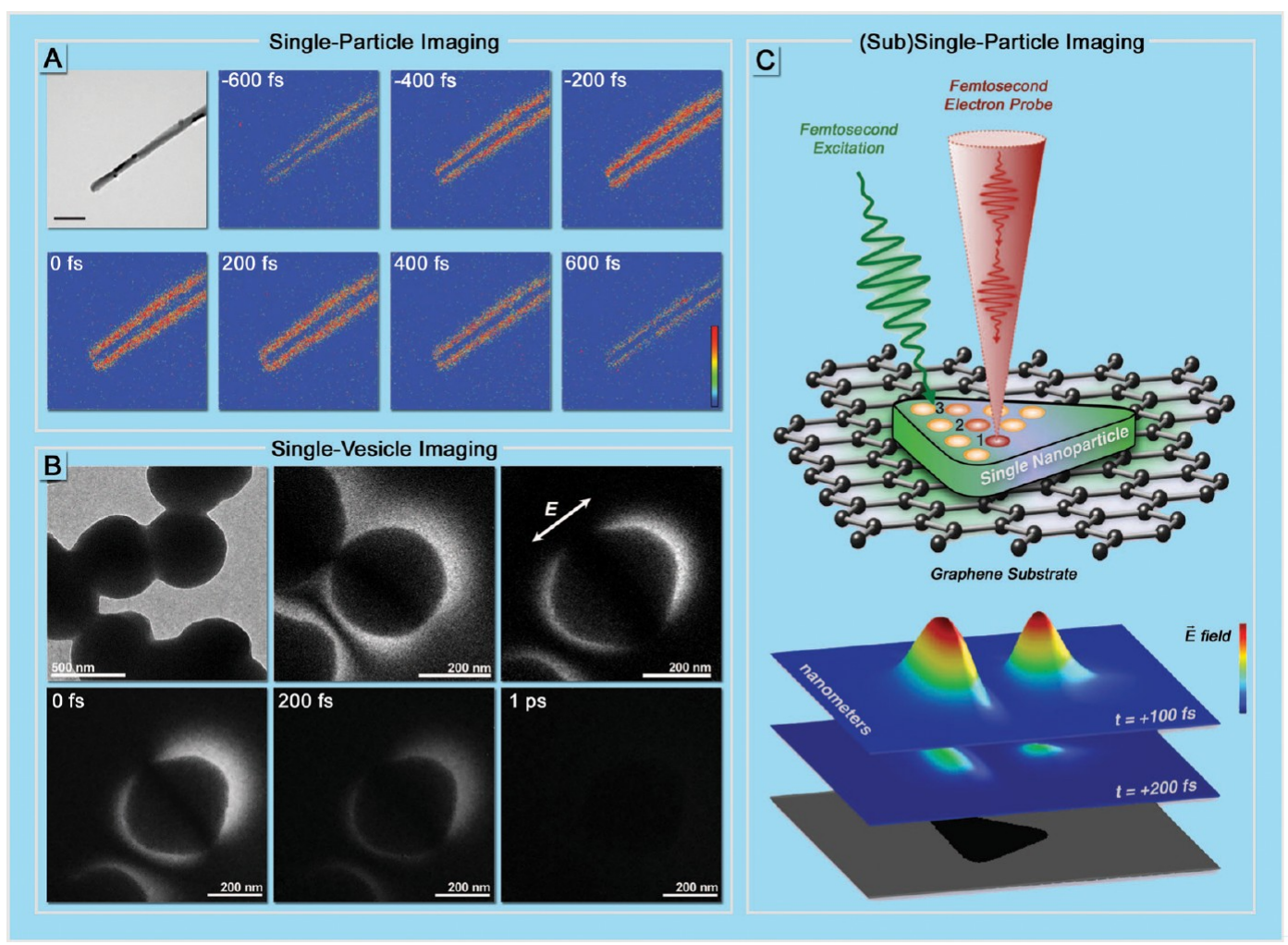

FIGURE 1. Single-particle imaging in space and time. (A) Imaging of the evanescent field surrounding a carbon nanotube resolved with femtosecond temporal resolution. A bright-field image is shown for comparison. (B) Imaging of individual protein vesicles. The upper panels show from left to right: a bright-field image of several vesicles, a PINEM image of a single vesicle, and the effects of optical polarization on the PINEM image. The bottom panels show PINEM images of a vesicle resolved with femtosecond temporal resolution. (C) Schematic of (sub)single-particle convergent-beam PINEM on a graphene substrate with corresponding femtosecond time-resolved images (100 and $200 \mathrm{fs}$ ) of the particle evanescent fields.

preserving the ultrafast temporal response of UEM, thus opening the door to various applications in elemental analysis as well as mapping of interfaces and plasmonics.

Tomographic Imaging. Typical TEM images are 2D projections. The third spatial dimension parallel to the electron beam is a convolution of all the structural information along that axis due to a large depth of field in the object plane. In order to resolve the information along this axis with high resolution, a tomographic tilt series is acquired, and the discrete spatial information is extracted from this set of images via reconstruction algorithms. In this way, the three spatial dimensions of a specimen can be resolved and mapped with a TEM. ${ }^{27-29}$ To visualize the dynamics, however, the fourth dimension of time must be integrated into the entire tomographic series. This is accomplished with UEM by treating each tilt angle as a separate time scan via application of the concepts discussed above. In this way, the high resolution of the three spatial dimensions can be augmented with the ultrafast temporal resolution. ${ }^{12}$

The upper panels of Figure 2 display the experimental configuration (A) and conceptual framework (B) of 4D electron tomography. A heating pulse initiates a structural change, and an electron packet is used to image the specimen at some time delay relative to the heating pulse. The third spatial dimension is resolved in UEM in the same manner as in static electron tomography; the specimen is imaged at a series of tilt angles, and the three-dimensional structure is reconstructed from the two-dimensional projections. In order to temporally resolve the dynamics, a tilt series is generated at each delay, and the structure is reconstructed at each step in order to visualize all four dimensions.

Figure $2 \mathrm{C}$ shows the results from a 4D electron tomography series of experiments conducted on a complex carbon nanotube "ring". By acquiring a series of tomograms at discrete time steps, the mechanical motion of the ring structure was resolved with the necessary temporal resolution. Remarkably, it was observed that the complex structures exhibit clear oscillatory motions after laser-driven heating, with Fourier analysis revealing $\mathrm{MHz}$ frequencies and image mapping showing nanometer amplitudes. The wide range of time scales accessible in these studies is 


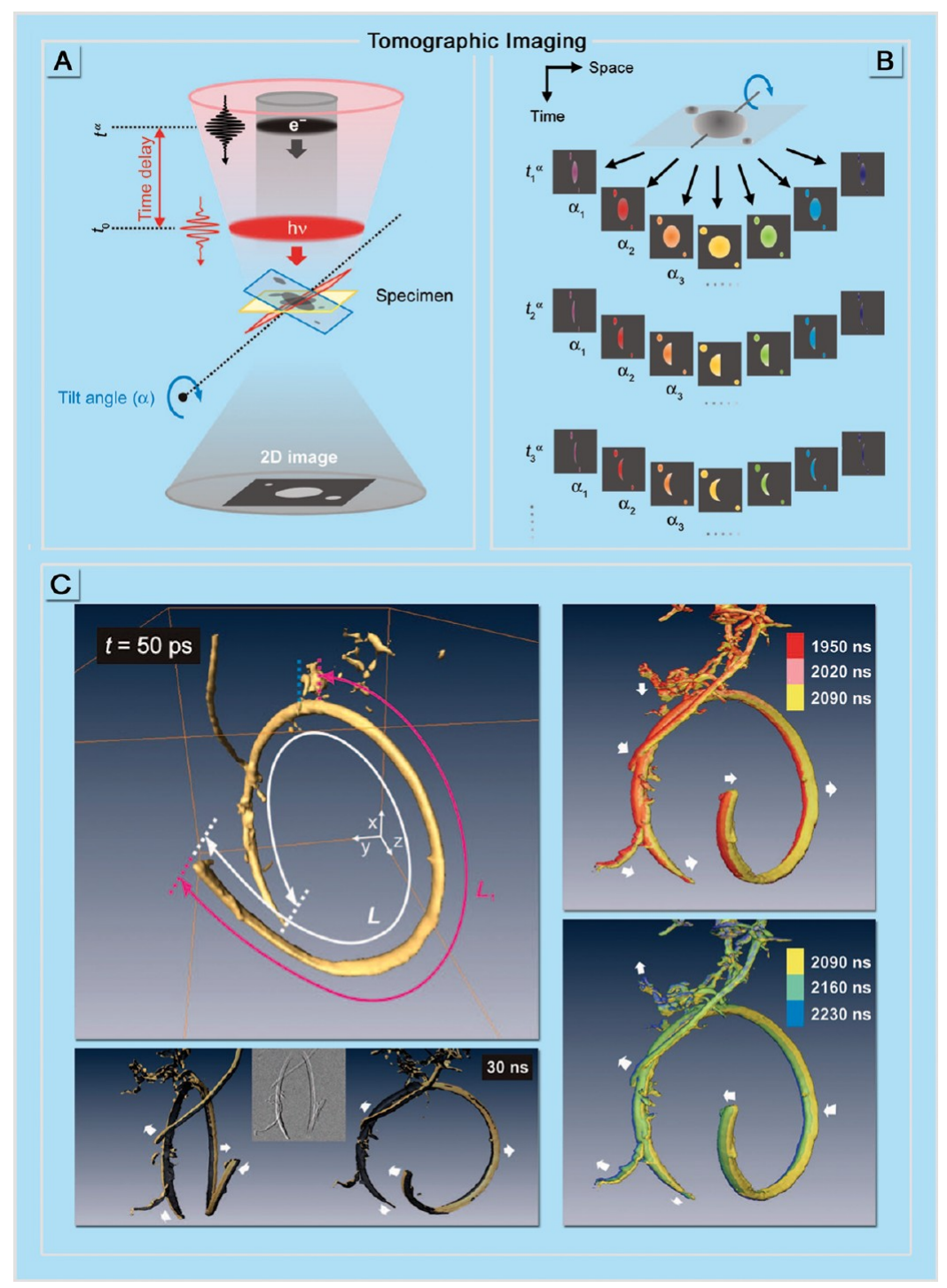

FIGURE 2. 4D electron tomography. (A) Schematic of 4D electron tomography. The key components of time delay and specimen tilting are illustrated. (B) Conceptual diagram of a series of images generated at various projection angles $\left(\alpha_{n}\right)$ and time steps $\left(t_{\mathrm{n}}{ }^{\alpha}\right)$ used to construct a $4 \mathrm{D}$ tomogram. (C) Tomographic images of freely suspended carbon nanotubes obtained at $50 \mathrm{ps}$ (upper left panel) and 30 ns (lower left panel). The lower left panel displays images obtained at two different angles, along with a difference image in the center. A reference image taken at $t=0 \mathrm{~ns}$ (black) is merged with the time-delayed image. White arrows indicate the direction of motion. Time-dependent structures visualized at microsecond delay times and with various colors and white arrows to illustrate the motion are shown to the right.

demonstrated in the panels, ranging from picoseconds, where electron-phonon coupling and phonon propagation are important, to microseconds and beyond, covering the regimes of resonance motions and diffusive heat dissipation.

Nanomechanical Motions. On the nanoscale, mechanical motions are exhibited in the time-resolved images and diffraction frames as the system executes such motions on the picosecond to millisecond scale. Examples studied include the motion of cantilevers, ${ }^{30}$ multiple cantilevers in nanomusical systems, ${ }^{31}$ the vibrational oscillations of graphite membranes, ${ }^{32,33}$ and the optomechanical friction motion at interfaces. ${ }^{34,35}$
The motion of a nanoscale object, when experiencing an applied force, depends upon dynamic processes that occur over a wide range of space and time. With UEM, we visualized the effects of interfacial forces on beams made of crystals of copper 7,7,8,8-tetracyanoquinodimethane $[\mathrm{Cu}(\mathrm{TCNQ})$; Figure $3 \mathrm{~A}]]^{30,34}$ The motions were induced by excitation with a pulsed laser, and the effects of interfacial forces on the dynamics were mapped out for two geometries: (1) crystals lying flat on a substrate and (2) crystals anchored at one end (cantilever). We found that the motion of the so-called fixed and free beams differed significantly due to substrate friction effects. By visualizing oscillations of 


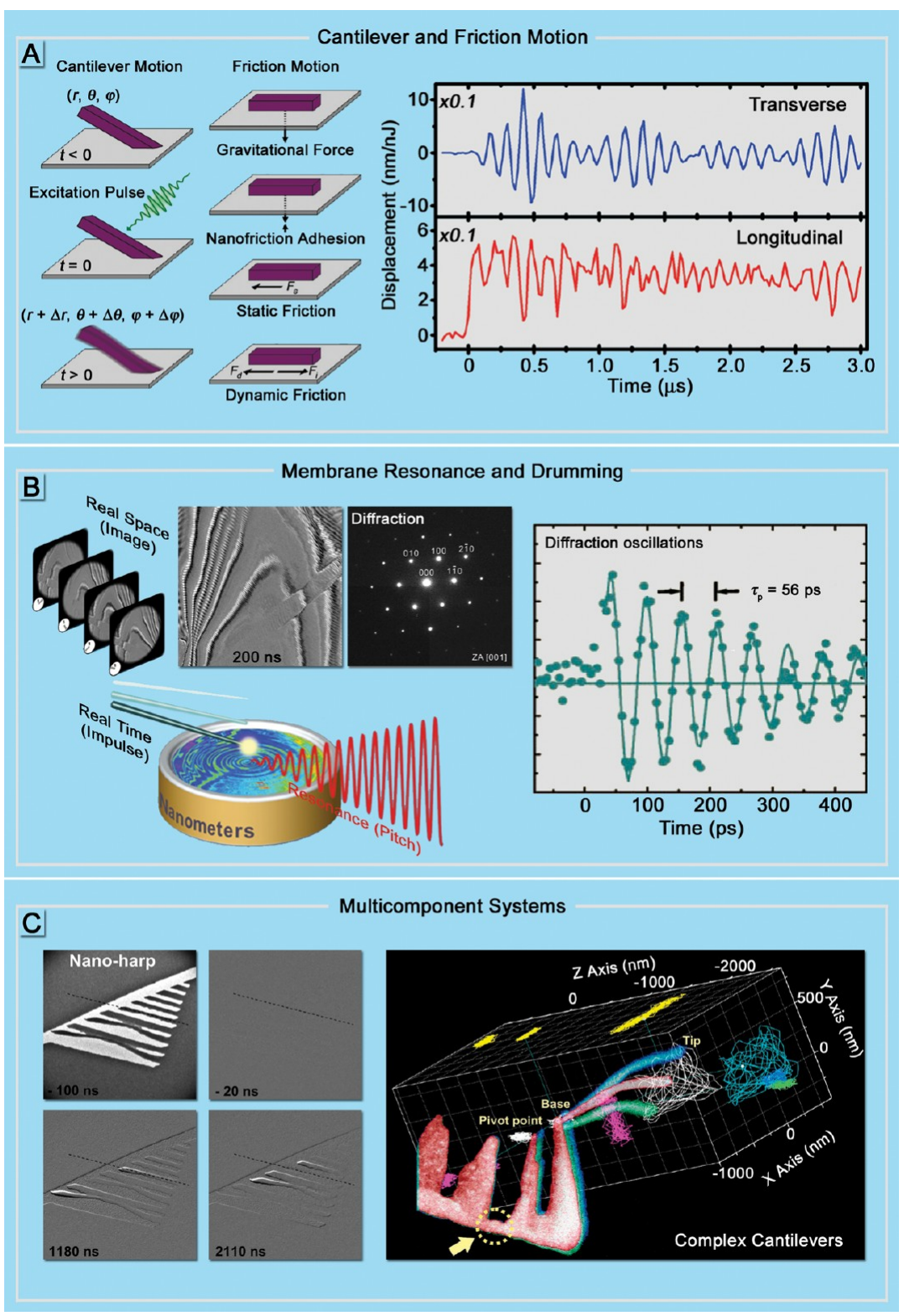

FIGURE 3. UEM of cantilevers and friction motion, membrane resonance and drumming, and multicomponent systems. (A) Cu(TCNQ) crystal cantilever and friction motion. After optical excitation of dynamics, structural changes of the crystals are mapped in real-space $(r, \theta, \varphi)$ and time $(t)$, and the various interfacial forces (adhesion, friction) present are determined. The transverse and longitudinal displacements of a cantilever crystal are plotted to the right. (B) Graphite membrane resonance and drumming. The oscillations are initiated impulsively with an optical pulse, and the resonance vibrations are resolved via UEM imaging. A representative difference image obtained at $200 \mathrm{~ns}$ is shown along with a single-crystal diffraction pattern. A plot of damped intensity oscillations of a Bragg spot in the diffraction pattern is shown to the right. The period is 56 ps. (C) Optomechanical motions of multicomponent systems. The four left panels show a bright-field and difference images of a "nano-harp". The frames are labeled with the times at which they were acquired relative to the excitation pulse. The right panel shows a three-dimensional plot of the ns "nano-arm" motion. Both nanodevices consisted of microfabricated nickel-titanium alloy.

the cantilever in real-space and time, we were able to observe resonant frequencies of a few $\mathrm{MHz}$. From this, the driving force and properties of the material were determined. For the fixed crystal, motion of the top and bottom surfaces of the fixed beam differed in both the temporal and spatial response to the optical pulse. From this motion, a static friction force of $0.5 \mu \mathrm{N}$ was determined, whereas a dynamic friction force of $0.4 \mu \mathrm{N}$ was found from the rate of steady sliding. The small load due to gravity $(120 \mathrm{fN})$ indicated that an additional effective load due to adhesion was largely responsible for the observed motion.

With UEM, it is also possible to study the nanoscale elasticity of materials. A prototype system is a membrane of graphite, which exhibits two types of structural dynamics, 
those that are longitudinal along a few nanometers thickness and those which are lateral and involve the entire membrane, as shown in Figure 3B. ${ }^{32,33}$ For this system, it was possible to determine the in-plane Young's modulus of 1.0 TPa and the perpendicular to the plane $c$-axis modulus of $30 \mathrm{GPa}$. The stress was introduced impulsively using the optical pulse while observing the motions using the stroboscopic electron pulses, from femtoseconds to milliseconds. After several microseconds, many of the modes are damped and the motion settles into a resonance of $1.1 \mathrm{MHz}$. Importantly, the information garnered from the UEM images is acquired without direct-contact probes. Further, the transients associated with the mechanical motion are resolved over a wide time range, spanning from picosecond oscillations, as observed in diffraction patterns, to microseconds and beyond for total vibrational damping.

A final example of visualization of nanomechanical motions is that involving "complex systems". They are made of various components, and their assembly motions display a whole range of frequencies. UEM enables the visualization of such systems because all components are imaged at once. Recently, we studied "nano-harp" and "nano-piano", which define "nanomusical" complex systems made of different "keys", either in their length or width or both; displayed in Figure $3 \mathrm{C}$ are the examples of "nano-harp" and "nanoarm". ${ }^{31,36}$ The frequencies involved were obtained using Fourier analysis and their knowledge permitted an excitation control of the "pitch". One fundamental issue here is the following: up to what nanoscale would the continuum classical models of materials properties breakdown?

Chemical Bonding and Reactions. As mentioned above, electron energy-loss spectroscopy (EELS) is a powerful technique capable of directly probing the electronic structure of materials. ${ }^{37}$ By measuring the change in energy of the primary electron beam due to scattering off the specimen, the nature of electronic structural change can be elucidated. The scope of applications spans surface and bulk elemental analysis, chemical characterization, and electronic structure determination. ${ }^{38}$ For studies of the dynamics of core and valence electronic structure and bonding, however, the time resolution must increase by 10 orders of magnitude. ${ }^{39}$ In femtosecond EELS (FEELS), the probing electrons and the optical excitation photons are both ultrashort, and the spectra can be recorded on this time scale of bonding. The concept of coherent single-electron packets is vital to this approach. When each ultrafast packet contains one electron on average, Coulomb repulsion is not a factor, and broadening of the electron energy distribution is absent.
The FEELS method was demonstrated in the study of graphite. ${ }^{13}$ By following the evolution of the energy spectra with femotosecond resolution, it was possible to resolve dynamic electronic changes with meV resolution (Figure 4A). Because spectra in this energy range contain information about valence and plasmon electrons, observed changes reveal the collective dynamics of bonding. Optical excitation of graphite generates charge-carriers which thermalize in $\sim 1 \mathrm{ps}$, and the FEELS results show that on this time scale there is a bond contraction due to an increase in threedimensional charge distribution. A sequence of dilations and expansions along the $c$-axis follows the compression and, at high laser fluence, strong interlayer distance variations occur. Thus, these observations reflect the change in electronic structure: contraction toward diamond and expansion toward graphene. ${ }^{13} \mathrm{~A}$ more recent study ${ }^{40}$ has shown that irradiation of graphite with fs pulses, not ns pulses, for a long time create tetrahedron-type structures, indeed consistent with FEELS findings.

Colossal structural changes may occur as a result of collective rearrangement of chemical bonding. Figure $4 \mathrm{~B}$ and $C$ shows the crystal structure and structural changes in real-time due to a laser-induced charge-transfer reaction in single $\mathrm{Cu}(\mathrm{TCNQ})$ crystals. ${ }^{41}$ This reaction occurs in the high laser fluence regime (Figure 4C), below the fluence used for the oscillatory motions discussed above, and whose manifestation in morphology change is evident (Figure 4B).

For the reaction, we observed the fast, initial structural rearrangement due to $\mathrm{Cu}^{+}$reduction, and the slower growth of metallic Cu nanocrystals following initiation of the reaction with a pulse of visible laser light:

$$
\left[\mathrm{Cu}^{+}\left(\mathrm{TCNQ}^{--}\right)\right]_{n} \stackrel{h v}{\rightarrow} \mathrm{Cu}_{x}^{0}+\mathrm{TCNQ}_{x}^{0}+\left[\mathrm{Cu}^{+}\left(\mathrm{TCNQ}^{\cdot-}\right)\right]_{n-x}
$$

From this and other numerous studies of $\mathrm{Cu}(\mathrm{TCNQ})$ made in this laboratory, the mechanism was determined to involve cooperative electron transfer from TCNQ anionradical to $\mathrm{Cu}^{+}$along the stacks, morphological changes, and thermally driven growth of the discrete metallic $\mathrm{Cu}$ nanocrystals. The entire process is complete in a few hundred microseconds.

T-jump, Thermal Expansions, and Phase Transitions. Allotropes of carbon are some of the most intensely studied materials owing to their remarkable mechanical, electronic, and thermal properties. The origin of these properties lies in the extended $\pi$-conjugation of the carbon network. Carbon nanotubes have unique structure and their thermal expansion may correlate with bonding characteristics, along the 


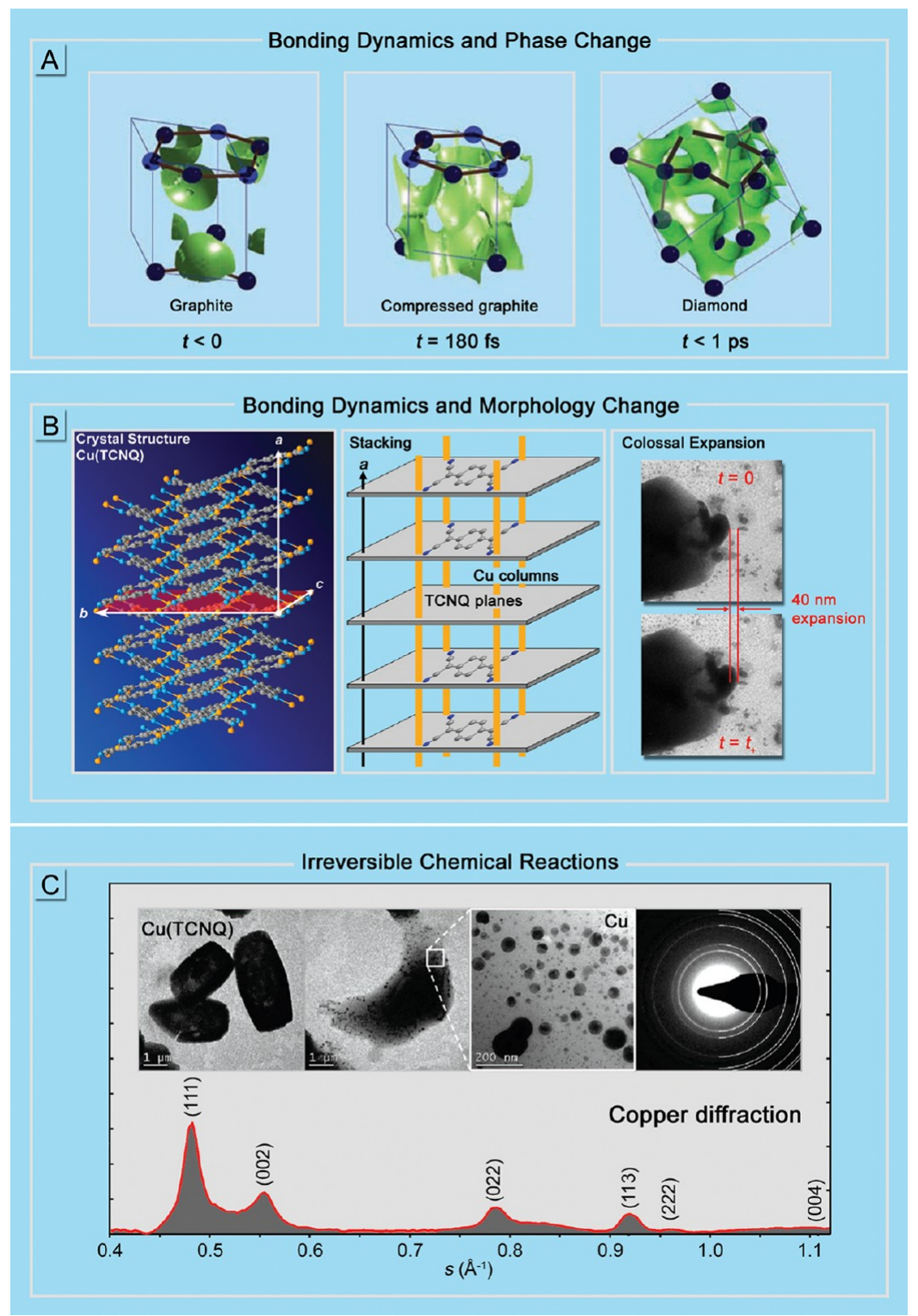

FIGURE 4. Bonding dynamics, phase and morphology change, and irreversible chemical reactions. (A) Femtosecond electron energy-loss spectroscopy (FEELS) of graphite. The three panels depict calculated charge density distributions for graphite $(c / a=2.7)$, compressed graphite $(c / a=$ 1.5), and diamond, the structures of which evolve on the femtosecond time scale. (B) Crystal structure (left panel) and schematic depiction (center panel) of the $\pi$-stacking morphology of $\mathrm{Cu}(\mathrm{TCNQ})$. The right panel illustrates the colossal nanometer crystal expansion after excitation. (C) Bright-field images and diffraction of the redox reaction producing Cu nanocrystals. Shown is the evolution from Cu(TCNQ) single crystals (left panel) to Cu metal as evidenced by diffraction of the nanostructures formed after laser excitation (right panel and indexed diffraction peak plot).

tube and perpendicular to it. With a T-jump, it was shown that the nonequilibrium lattice expansion of carbon nanotubes on the picosecond to millisecond time scales (Figure $5 \mathrm{~A}$ ) is highly anisotropic. ${ }^{42}$ The time-resolved diffraction, following ultrafast heating at the rate of $10^{13} \mathrm{~K} / \mathrm{s}$, showed that the nanotube expands only in the radial (intertubule) direction, whereas no significant change was observed in the intratubular axial or equatorial dimensions. Here, the value in using diffraction resides in the identification of the different atomic planes as shown in Figure 5A.
The recovery time, which represents the heat dissipation rate for equilibration, and occurs on slower time scales, was found to be on the order of $\sim 100 \mu \mathrm{s}$, and this recovery was reproduced theoretically by considering the composite specimen-substrate heat exchange properties.

At relatively high temperatures, one can examine phase transitions, and here we discuss two cases: phase changes involved in nucleation and crystallization in silicon and the phase transition of martensitic type in iron. The transformation of a structure from amorphous to crystalline involves 

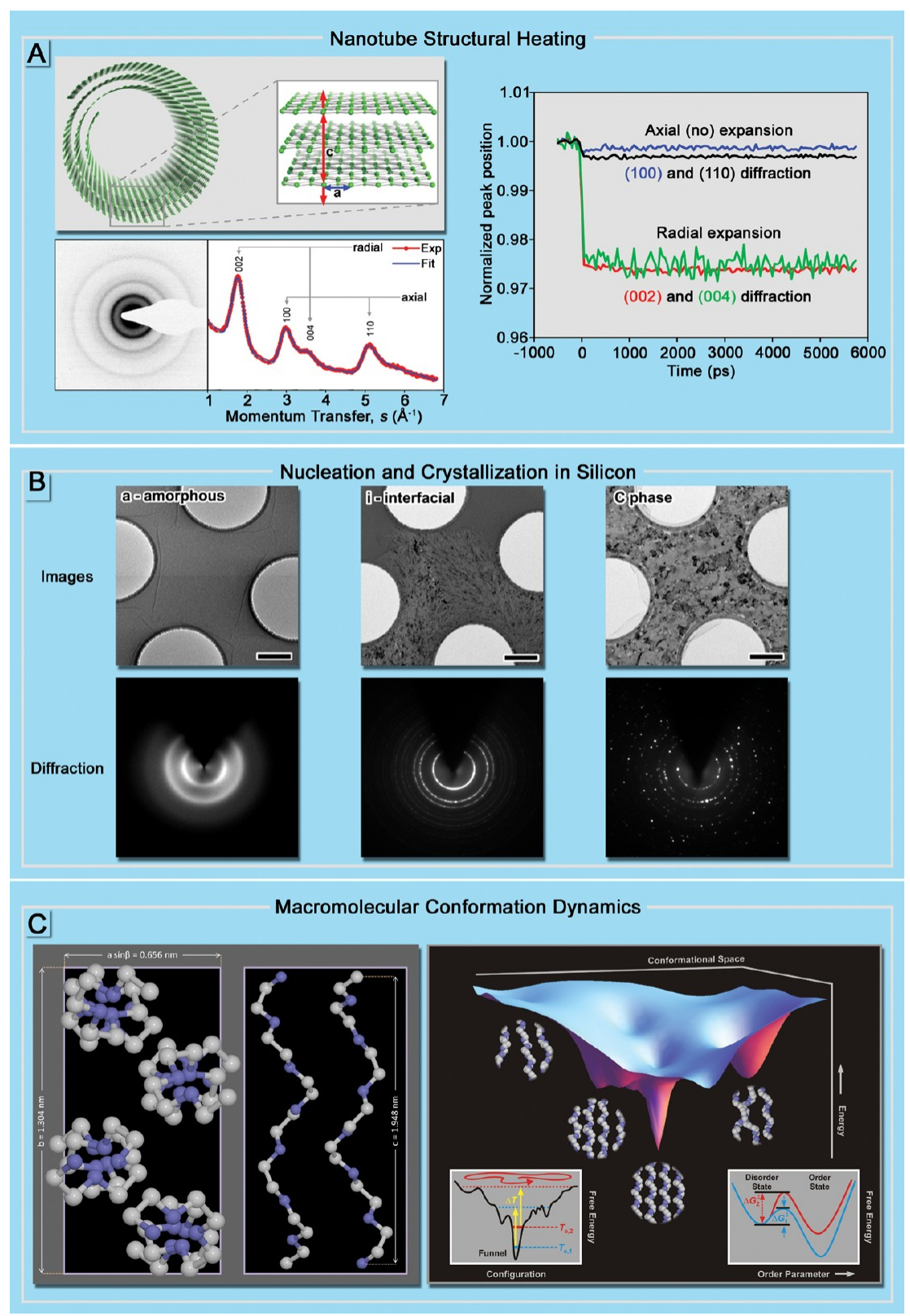

FIGURE 5. Nanotube structural heating, nucleation and crystallization of silicon, and macromolecular conformation dynamics. (A) Anisotropic expansion of carbon scrolls. Schematic of a carbon scroll and anisotropic expansion is shown in the upper left panel. Below this is displayed the Debye-Scherrer ring pattern and radially integrated curve with fit. The peaks are assigned and categorized as radial or axial in orientation. The right panel shows the anisotropic ps dynamics of carbon scrolls. The axial (intratubule) directions [(100) and (110)] show no expansion, while the radial (intertubule) directions [(002) and (004)] show large, picosecond expansion. (B) Nucleation dynamics of silicon. The response after single laser-pulse heating is shown. The zones are amorphous (a), interfacial (i), and crystalline (C) phases. Shown below the images are diffraction patterns of the (a), (i), and $(\mathrm{C})$ regions. $(\mathrm{C})$ Macromolecular conformation dynamics. The left panel shows the PEO monoclinic unit cell as projected onto a plane perpendicular to the $c$-axis with a side view of left- and right-handed helices. The free-energy landscape in molecular conformation space is shown to the right. For this representation, the entropy is reflected in the width whereas the enthalpy is given by the depth. Also displayed are pictorial snapshots for representative macromolecular conformations. The inset displays simplified free energy surfaces showing (left) the effect of initial equilibrium temperature when a T-jump experiment is carried out, and (right) the order/disorder two-state representation as the order parameter changes.

complex dynamic processes occurring over a wide range of energy, length, and time. Elucidation of the steps involved requires direct visualization of the irreversible process with the proper spatiotemporal resolution. Using the single-shot mode of imaging, the process of phase transformation of amorphous silicon, through the liquid phase to crystalline phase (Figure $5 \mathrm{~B}),{ }^{43}$ was visualized. The T-jump was used to initiate crystallization from the amorphous phase, while a 
single pulse of electrons was used to image the transformation. With selected-area diffraction, the amorphous and crystalline phases were identified, and the temporal profile of the phase transformation was obtained. Initiation via nanoscale surface melting occurs within $10 \mathrm{ps}$, but transformation to the crystalline state takes much longer. On this time scale, there is no significant heat diffusion within the probed volume, and two main processes were identified: quasi-coherent nanoscale layer propagation at $\sim 15 \mathrm{~m} / \mathrm{s}$, and another that occurs with much slower velocity.

Unlike the case of amorphous-to-crystalline phase change, martensitic transformations occur via a diffusionless process with small reorientations of atom positions in the crystal lattice. The associated time and length scales of such transformations often limit direct microscopic observation of the process. Real-time martensitic phase transformation of Fe from a bcc to fcc crystal structure (Figure $5 \mathrm{C})^{44}$ was similarly studied using the T-jump heating at $\sim 10^{11} \mathrm{~K} / \mathrm{s}$, and the evolution of the phase transformation was followed using selected-area diffraction. Two distinct transient components were revealed in the evolution of the crystal structure: (1) a direct martensitic transformation occurring on the nanosecond time scale, which proceeds in regions heated into the temperature range within which the fcc phase is stable, and (2) the microsecond time scale representing an indirect process for the hottest central zone of laser heating. The studies indicate that the mechanism involves two steps, a barrier-crossing nucleation on the nanosecond time scale followed by rapid grain growth in $\sim 100$ ps for $10 \mathrm{~nm}$ crystallites.

\section{Emerging Developments}

4D Imaging with Scanning Probes: S-UEM and ST-UEM. With scanning electron microscopy (SEM), it is possible to generate images of the surface features of nanostructures. Importantly, the physics associated with image formation in the SEM, coupled with low accelerating voltages, make studies in ambient conditions amenable. Scanning ultrafast electron microscopy (S-UEM) is uniquely suited for the probing of surface structures in real-space and time. ${ }^{15,45}$ So far, the focus has been on the dynamics of surfaces under vacuum conditions, but recently the technique was extended to study solvation at interfaces between semiconductors and polar molecules such as water and acetonitrile. It is found that surface polarity, as determined by its orientation, has a critical role in determining the time scale of solvation and surface-solvent interactions at the nanometer interfacial scale. $^{46}$
Unlike S-UEM, which depends on the generation of secondary electrons for imaging, the technique of scanning transmission ultrafast electron microscopy (ST-UEM) utilizes the transmitted electrons through the specimen. One advantage is that electrons scattered at high angles, which leads to strong atomic number $\left(\mathrm{Z}^{2}\right)$-dependent contrast, enables a straightforward interpretation of the image. Importantly, nanoscale regions of a specimen can be imaged while simultaneously collecting electron energy-loss information from that particular region. For example, for silver nanowires, an ST-UEM movie vividly illustrates the mechanical motion of the cantilever-type object, and the frequency and damping rate of the structural vibrations were determined directly from the time-resolved frames. From the same nanometer probed region, electron spectra were obtained. These scanning probes with UEM capabilities offer new dimensions in the study of structural dynamics.

Polymers and Biological Imaging. Macromolecular structural dynamics, unlike those of small molecules and elemental materials, involve complex free-energy landscapes with numerous possible conformations across a wide range of time scales. A prime example is that of protein folding that occurs as a result of a search from a high-entropy state of many conformations to the low-entropy native structure. ${ }^{47}$ The folding behavior has been likened to a phase transition, and in this regard the concepts are related to glass transitions and to other macromolecular systems such as polymers. 4D electron microscopy was recently applied to visualize the macromolecular dynamics of helical poly(ethylene oxide) (PEO), a model system, using diffraction and dark-field imaging. ${ }^{48}$ One of the most important findings is pertinent to the nature of the free energy landscape. By varying the nonequilibrium temperature jump, the effect of initial equilibrium temperature, and the time scale for the loss of order for different orientations and interchain separations, it was possible to understand the entropic bottleneck in the funnel description of the free-energy landscape and to experimentally demonstrate the conformational changes involved. Clearly, the next step is a study of protein conformations. $^{49}$

So far, with UEM, imaging has been achieved for vesicles, as discussed above, and for crystals and cells. ${ }^{6,7}$ But more needs to be advanced regarding studies of systems under their ambient conditions. The advent of solution cells ${ }^{50}$ and environmental chambers for $\mathrm{TEM}^{51}$ has opened up new vistas for high-resolution imaging of live cells and liquidphase chemical processes such as crystal growth, diffusion, and heterogeneous reactions. By combining these specimen 
stages with the UEM method, the origin of the dynamic processes occurring under such conditions can be probed beyond the millisecond temporal range afforded by current TEM CCD cameras. Indeed, there is much interest in elucidating the first moments of biochemical processes vital to cellular functions, the reactions of which occur on the picosecond time scales. In addition, the mechanisms for nanocrystal growth, coalescence, and passivation via oxidation must be probed under the proper synthetic conditions and with the necessary temporal resolution. These and a wide range of other systems are well-suited for study with UEM at or near ambient conditions.

\section{Concluding Remarks}

The electron microscope is a powerful scientific instrument due to the ability to comprehensively characterize inorganic, organic, and biological systems with unparalleled spatial resolution, the atomic scale. The integration of the fourth dimension-time-now enables for the vast nonequilibrium space of dynamics to be visualized, and with the same spatial resolution. The opportunities are numerous. The advent of near-field and convergent beam 4D UEM variants opens the door for applications in nanoscale plasmonics and photonics and for studies of heterogeneous processes, such as those involved in the catalytic properties of nanoparticles or in phase transitions. With recent developments in attosecond optical pulses ${ }^{52}$ and schemes for attosecond electron pulses, ${ }^{21,22}$ we may soon make possible, with UEM, the direct imaging of electron dynamics. What is certain is that $4 \mathrm{D}$ electron microscopy will continue applications in materials science; it has already matured as a method with different variants. Similarly, we anticipate further exploration into the biological imaging world.

This research was supported by the National Science Foundation, the Air Force Office of Scientific Research, and the Gordon and Betty Moore Foundation. We would like to express our sincere gratitude to members of our laboratories, past and present, who contributed significantly to the research discussed here; their contributions are acknowledged in the publications referenced here.

\section{BIOGRAPHICAL INFORMATION}

David J. Flannigan is the Ray D. and Mary T. Johnson/Mayon Plastics Assistant Professor of Chemical Engineering and Materials Science at the University of Minnesota. Prior to joining the faculty at Minnesota in the summer of 2012, he was a senior postdoctoral scholar at Caltech where he worked, with Prof. Zewail, on ultrafast electron microscopy. He received a B.S. in Chemistry from the University of Minnesota and a Ph.D. in Chemistry under the guidance of Prof. Ken Suslick at the University of Illinois at Urbana-Champaign where he studied the chemical and physical conditions generated during acoustic cavitation.

Ahmed H. Zewail is the Linus Pauling Professor of Chemistry and Professor of Physics, and Director of the Physical Biology Center at Caltech. He received the 1999 Nobel Prize in Chemistry for his development of "Femtochemistry", making possible observations of transition states and ephemeral molecular phenomena on the femtosecond $\left(10^{-15}\right.$ second) time scale. More recently, he and his group have developed the field of " $4 D$ electron microscopy" imaging, enabling visualization of structures in both space and time. In 2009, he was appointed by President Barack Obama to the President's Council of Advisors on Science and Technology. He also became the United States' first Science Envoy.

\section{FOOTNOTES}

*To whom correspondence should be addressed. Phone: (+1) 626-395-6536. Fax: $(+1)$ 626-792-8456. E-mail: zewail@caltech.edu.

The authors declare no competing financial interest.

${ }^{t}$ Department of Chemical Engineering and Materials Science, University of Minnesota, Minneapolis, MN 55455, USA.

\section{REFERENCES}

1 Bragg, W. L. The Structure of Some Crystals as Indicated by their Diffraction of X-rays. Proc. R. Soc. London, Ser. A 1913, 89, 248-277.

2 Klinge, S.; Voigts-Hoffmann, F.; Leibundgut, M.; Arpagaus, S.; Ban, N. Crystal Structure of the Eukaryotic 60S Ribosomal Subunit in Complex with Initiation Factor 6. Science 2011, 334, 941-948.

3 Henke, B. L.; Gullikson, E. M.; Davis, J. C. X-ray Ineractions: Photoabsorption, Scattering, Transmission, and Reflection at $\mathrm{E}=50-30,000 \mathrm{eV}, \mathrm{Z}=1-92$. At. Data Nucl. Data Tables 1993, 54, 181-342.

4 Riley, M. E.; MacCallum, C. J.; Biggs, F. Theoretical Electron-Atom Elastic Scattering Cross Sections: Selected Elements, 1 to 256 keV. At. Data Nucl. Data Tables 1975, 15, 443476.

5 Zewail, A. H. Femtochemistry: Atomic-Scale Dynamics of the Chemical Bond Using Ultrafast Lasers. Angew. Chem., Int. Ed. 2000, 39, 2587-2631 (the 1999 Nobel Lecture).

6 Zewail, A. H.; Thomas, J. M. 4D Electron Microscopy: Imaging in Space and Time; Imperial College Press: London, 2010.

7 Zewail, A. H. Four-Dimensional Electron Microscopy. Science 2010, 328, 187-193.

8 Shorokhov, D.; Zewail, A. H. 4D Electron Imaging: Principles and Perspectives. Phys. Chem. Chem. Phys. 2008, 10, 2879-2893.

9 Shorokhov, D.; Zewail, A. H. New Light on Molecular and Materials Complexity: 4D Electron Imaging. J. Am. Chem. Soc. 2009, 131, 17998-18015.

10 Yurtsever, A.; Zewail, A. H. 4D Nanoscale Diffraction Observed by Convergent-Beam Ultrafast Electron Microscopy. Science 2009, 326, 708-712.

11 Barwick, B.; Flannigan, D. J.; Zewail, A. H. Photon-Induced Near-Field Electron Microscopy. Nature 2009, 462, 902-906.

12 Kwon, 0.-H.; Zewail, A. H. 4D Electron Tomography. Science 2010, 328, 1668-1673.

13 Carbone, F.; Kwon, 0.-H.; Zewail, A. H. Dynamics of Chemical Bonding Mapped by Energy-Resolved 4D Electron Microscopy. Science 2009, 325, 181-184.

14 Park, H. S.; Baskin, J. S.; Zewail, A. H. 4D Lorentz Electron Microscopy Imaging: Magnetic Domain Wall Nucleation, Reversal, and Wave Velocity. Nano Lett. 2010, 10, 3796-3803.

15 Yang, D.-S.; Mohammed, O. F.; Zewail, A. H. Scanning Ultrafast Electron Microscopy. Proc. Natl. Acad. Sci. U.S.A. 2010, 107, 14993-14998.

16 Ortalan, V.; Zewail, A. H. 4D Scanning Transmission Ultrafast Electron Microscopy: SingleParticle Imaging and Spectroscopy. J. Am. Chem. Soc. 2011, 133, 10732-10735.

17 Baskin, J. S.; Zewail, A. H. Freezing Atoms in Motion: Principles of Femtochemistry and Demonstration by Laser Stroboscopy. J. Chem. Educ. 2001, 78, 737-751.

18 Browning, N. D.; Bonds, M. A.; Campbell, G. H.; Evans, J. E.; LaGrange, T.; Jungjohann, K. L.; Masiel, D. J.; McKeown, J.; Mehraeen, S.; Reed, B. W.; Santala, M. Recent Developments in Dynamic Transmission Electron Microscopy. Curr. Opin. Solid State Mater. Sci. 2012, 16, 23-30. 
19 Zewail, A. H.: Lobastov, V. A. Method and System for Ultrafast Photoelectron Microscope. U. S. Patent 7,154,091, Dec. 26, 2006.

20 Thomas, J. M. A Revolution in Electron Microscopy. Angew. Chem., Int. Ed. 2005, 44, 5563-5566.

21 Baum, P.; Zewail, A. H. Attosecond Electron Pulses for 4D Diffraction and Microscopy. Proc. Natl. Acad. Sci. U.S.A. 2007, 104, 18409-18414.

22 Hilbert, S. A.; Uiterwaal, C.; Barwick, B.; Batelaan, H.; Zewail, A. H. Temporal Lenses for Attosecond and Femtosecond Electron Pulses. Proc. Natl. Acad. Sci. U.S.A. 2009, 106, 10558-10563.

23 Zewail, A. H. 4D Ultrafast Electron Diffraction, Crystallography, and Microscopy. Annu. Rev. Phys. Chem. 2006, 57, 65-103.

24 Flannigan, D. J.; Barwick, B.; Zewail, A. H. Biological Imaging with 4D Ultrafast Electron Microscopy. Proc. Natt. Acad. Sci. U.S.A. 2010, 107, 9933-9937.

25 Park, S. T.; Lin, M.; Zewail Ahmed, H. Photon-Induced Near-Field Electron Microscopy (PINEM): Theoretical and Experimental. New J. Phys. 2010, 12, 123028.

26 Yurtsever, A.; van der Veen, R. M.; Zewail, A. H. Subparticle Ultrafast Spectrum Imaging in 4D Electron Microscopy. Science 2012, 335, 59-64.

27 Friedrich, H.; de Jongh, P. E.; Verkleij, A. J.; de Jong, K. P. Electron Tomography for Heterogeneous Catalysts and Related Nanostructured Materials. Chem. Rev. 2009, 109, 1613-1629.

28 Frank, J. Electron Tomography: Methods for Three-Dimensional Visualization of Structures in the Cell; Springer: New York, 2006.

29 Midgley, P. A.; Ward, E. P. W.; Hungria, A. B.; Thomas, J. M. Nanotomography in the Chemical, Biological, and Materials Sciences. Chem. Soc. Rev. 2007, 36, 14771494.

30 Flannigan, D. J.; Samartzis, P. C.; Yurtsever, A.; Zewail, A. H. Nanomechanical Motions of Cantilevers: Direct Imaging in Real Space and Time with 4D Electron Microscopy. Nano Lett. 2009, 9, 875-881.

31 Baskin, J. S.; Park, H. S.; Zewail, A. H. Nanomusical Systems Visualized and Controlled in 4D Electron Microscopy. Nano Lett. 2011, 11, 2183-2191.

32 Barwick, B.; Park, H. S.; Kwon, 0.-H.; Baskin, J. S.; Zewail, A. H. 4D Imaging of Transient Structures and Morphologies in Ultrafast Electron Microscopy. Science 2008, 322, 12271231.

33 Kwon, 0.-H.; Barwick, B.; Park, H. S.; Baskin, J. S.; Zewail, A. H. Nanoscale Mechanical Drumming Visualized by 4D Electron Microscopy. Nano Lett. 2008, 8, 3557-3562.

34 Flannigan, D. J.; Park, S. T.; Zewail, A. H. Nanofriction Visualized in Space and Time by 4D Electron Microscopy. Nano Lett. 2010, 10, 4767-4773.

35 Flannigan, D. J.; Zewail, A. H. Optomechanical and Crystallization Phenomena Visualized with 4D Electron Microscopy: Interfacial Carbon Nanotubes on Silicon Nitride. Nano Lett. 2010, 10, 1892-1899.
36 Kwon, 0.-H.: Park, H. S.; Baskin, J. S. Z Zewail, A. H. Nonchaotic Nonlinear Motion Visualized in Complex Nanostructures by Stereographic 4D Electron Microscopy. Nano Lett. 2010, 10, 3190-3198.

37 Egerton, R. F. Electron Energy-Loss Spectroscopy in the Electron Microscope; Springer: New York, 2011

38 Thomas, J. M.; Williams, B. G.; Sparrow, T. G. Electron-Energy-Loss Spectroscopy and the Study of Solids. Acc. Chem. Res. 1985, 18, 324-330.

39 Thomas, J. M. The Renaissance and Promise of Electron Energy-Loss Spectroscopy. Angew. Chem., Int. Ed. 2009, 48, 8824-8826.

40 Kanasaki, J.; Inami, E.; Tanimura, K.; Ohnishi, H.; Nasu, K. Formation of sp ${ }^{3}$-Bonded Carbon Nanostructures by Femtosecond Laser Excitation of Graphite. Phys. Rev. Lett. 2009, 102, 087402.

41 Park, S. T.; Flannigan, D. J.; Zewail, A. H. Irreversible Chemical Reactions Visualized in Space and Time with 4D Electron Microscopy. J. Am. Chem. Soc. 2011, 133, 1730-1733.

42 Park, S. T.; Flannigan, D. J.; Zewail, A. H. 4D Electron Microscopy Visualization of Anisotropic Atomic Motions in Carbon Nanotubes. J. Am. Chem. Soc. 2012, 134, 9146-9149.

43 Kwon, 0.-H.; Barwick, B.; Park, H. S.; Baskin, J. S.; Zewail, A. H. 4D Visualization of Embryonic, Structural Crystallization by Single-Pulse Microscopy. Proc. Natt. Acad. Sci. U.S.A. 2008, 105, 8519-8524.

44 Park, H. S.; Kwon, 0.-H.; Baskin, J. S.; Barwick, B.; Zewail, A. H. Direct Observation of Martensitic Phase-Transformation Dynamics in Iron by 4D Single-Pulse Electron Microscopy. Nano Lett. 2009, 9, 3954-3962.

45 Mohammed, 0. F.; Yang, D.-S.; Pal, S. K.; Zewail, A. H. 4D Scanning Ultrafast Electron Microscopy: Visualization of Materials Surface Dynamics. J. Am. Chem. Soc. 2011, 133, 7708-7711.

46 Yang, D.-S.; Mohammed, 0. F.; Zewail, A. H. Environmental Scanning Ultrafast Electron Microscopy: Structural Dynamics of Solvation at Interfaces (submitted for publication in Angew. Chem.)

47 Onuchic, J. N.; Luthey-Schulten, Z.; Wolynes, P. G. Theory of Protein Folding: The Energy Landscape Perspective. Annu. Rev. Phys. Chem. 1997, 48, 545-600.

48 Kwon, 0.-H.; Ortalan, V.; Zewail, A. H. Macromolecular Structural Dynamics Visualized by Pulsed Dose Control in 4D Electron Microscopy. Proc. Natl. Acad. Sci. U.S.A. 2011, 108, 6026-6031.

49 Zewail, A. H. Filming the Invisible in 4-D. Sci. Am. 2010, 303, 74-81.

50 de Jonge, N.; Ross, F. M. Electron Microscopy of Specimens in Liquid. Nat. Nanotechnol. 2011, 6, 695-704.

51 Gai, P. L.; Boyes, E. D. Advances in Atomic Resolution In Situ Environmental Transmission Electron Microscopy and 1 A Aberration Corrected In Situ Electron Microscopy. Microsc. Res. Tech. 2009, 72, 153-164.

52 Corkum, P. B.; Krausz, F. Attosecond Science. Nat. Phys. 2007, 3, 381-387. 